\title{
Variations of Hawk Mimicry Traits in the Four Sympatric Cuculus Cuckoos
}

\author{
Jun-Seo Go, Jin-Won Lee* and Jeong-Chil Yoo \\ Department of Biology, Korea Institute of Ornithology, Kyung Hee University, Seoul, South Korea
}

A well-known visual signal, hawk-like features such as yellow eyes and feet, and barred underparts have been recognized as coevolutionary traits obtained against host defense in Cuculus cuckoos. However, the variation of these traits within and among species remains poorly understood because empirical studies quantifying these traits are limited in terms of the number of studies and the number of species concerned, and mostly depend on museum collections. In this study, we quantified and compared these traits as well as other new features (e.g., inner wing spot and underpart background color) in the four sympatric Cuculus cuckoos (Cuculus poliocephalus, Cuculus micropterus, Cuculus optatus, and Cuculus canorus) that were wild-captured in South Korea. We found that the yellow color of the eye ring and feet was fairly consistent across the four species. However, the iris color appeared to vary within a species (e.g., between sexes) and varied more substantially among species from nearly black in C. micropterus to bright yellow in $C$. canorus. In addition, there were significant differences among species with respect to the thickness of the underpart bars, from the thinnest in $C$. canorus to

OPEN ACCESS

Edited by:

Canchao Yang,

Hainan Normal University, China

Reviewed by:

Rose Thorogood, University of Helsinki, Finland

Canwei Xia,

Beijing Normal University, China

*Correspondence:

Jin-Won Lee

jwlee99@khu.ac.kr

Specialty section:

This article was submitted to

Coevolution,

a section of the journal

Frontiers in Ecology and Evolution

Received: 29 April 2021

Accepted: 21 July 2021

Published: 06 August 2021

Citation:

Go J-S, Lee J-W and Yoo J-C (2021) Variations of Hawk Mimicry

Traits in the Four Sympatric Cuculus Cuckoos. Front. Ecol. Evol. 9:702263.

doi: 10.3389/fevo.2021.702263 the thickest in C. micropterus. We also found that the underpart color (pure white versus yellowish brown) and the number of inner wing spots varied within and among species. These results indicate that although hawk-like traits are widely present in Cuculus cuckoos, detailed quantitative features of these traits vary across species. We discuss the potential reasons that generate such variations and suggest future directions to increase our understanding of visual signals in avian brood parasitism.

Keywords: brood parasitism, Cuculus cuckoos, hawk mimicry, morphology, reciprocal interactions

\section{INTRODUCTION}

Direct, swift flight with a long tail and wings, an elongated body with gray upperparts and pale barred underparts, and yellow eyes and yellow legs, are all features that characterize raptors such as Accipiter hawks (Parkes, 1955; Kuroda, 1966; Payne, 1967; Newton and Marquiss, 1982; Duckworth, 1991; Davies and Welbergen, 2008; Newton, 2010; Welbergen and Davies, 2011). Interestingly, however, these features are also observed in the common cuckoo, Cuculus canorus, a brood parasite that lays its eggs in the nests of other species, namely the host, which provides parental care such as incubation and feeding of its progeny (Friedmann, 1928; Payne, 1977; Rothstein, 1990; Davies, 2000; Payne and Sorensen, 2005; Erritzøe et al., 2012; Medina and Langmore, 2016). People have been intrigued by such cuckoo-hawk resemblances, and many hypotheses have been proposed to explain the adaptive function of hawk mimicry by cuckoos (Wallace, 1889; Kuroda, 1966; Davies and Welbergen, 2008). For example, Wallace (1889) suggested that hawk mimicry may reduce attacks from predators, such as hawks, on cuckoos that need to spend a substantial amount of time on a perch to observe hosts. Alternatively, Davies and Welbergen (2008) suggested that hawks and cuckoos may have independently evolved such plumage patterns for a cryptic function of 
countershading, thereby reducing the chances of detection by their prey or hosts. However, the most accepted hypothesis proposed to date is that hawk mimicry is Batesian mimicry that has evolved in cuckoos to increase the chance of accessing host nests while escaping host aggression, by mimicking their potential predators, such as sparrowhawks Accipiter nisus and kestrels Falco tinnunculus (for the rufous morphs) (Voipio, 1953; Kuroda, 1966; Welbergen and Davies, 2011; Gluckman and Mundy, 2013; Thorogood and Davies, 2013). In other words, hawk mimicry is understood as a coevolutionary outcome of reciprocal interactions between cuckoos and hosts, as best seen in host egg mimicry (Welbergen and Davies, 2011).

Empirical studies to date that have experimentally tested the adaptive function of hawk mimicry, especially like underpart barring, have generally led to inconsistent results with respect to host species and populations, triggering either fear or aggression as a response (Duckworth, 1991; Honza et al., 2006; Welbergen and Davies, 2009, Welbergen and Davies, 2011; Trnka and Prokop, 2012; Trnka et al., 2012; Trnka and Grim, 2013; Ma et al., 2018). For example, some host species, such as the reed warbler Acrocephalus scirpaceus and some nonhost species, including the great tit Parus major and blue tit Cyanistes caeruleus, showed escape responses to the exposure of cuckoo dummy and the sparrowhawk dummy, where the presence of underpart barring played a significant role (Davies and Welbergen, 2008; Welbergen and Davies, 2011). However, in the great reed warbler Acrocephalus arundinaceus, which is another aggressive host species of the common cuckoo, aggression instead of such escape responses, was observed (Dyrcz and Hałupka, 2006; Honza et al., 2006; Trnka and Prokop, 2012; Trnka et al., 2012). The relative costs and benefits of host responses that are determined, for example, by the presence of model species, that is, dangerous hawks, and/or their local density may generate such variations (Trnka and Grim, 2013). Given that mimicry emerged as a counteracting strategy for host discrimination, individual variation of the degree of hawk mimicry by cuckoos (i.e., intra-specific variation of hawk mimicry) and variations in host responses to such hawk mimicry may be an underlying cause of the generation of mimicry dynamics across cuckoo host races and populations (Davies and Welbergen, 2009; Welbergen and Davies, 2009). In other words, as the degree of host egg mimicry increases in response to increasing host egg rejection, a higher degree of hawk mimicry is achieved in cuckoo host races or populations that undergo higher degrees of host discrimination (Davies and Welbergen, 2009; Welbergen and Davies, 2009). Furthermore, such hawk-like features as underpart barring and yellow eyes occur as a result of Batesian mimicry not only in the common cuckoo but also in other species belonging to different genera (e.g., Chrysococcyx, Eudynamys, Cacomantis, and Cerococcyx) of old world parasitic cuckoos (Gluckman and Mundy, 2013; Thorogood and Davies, 2013). Through comprehensive comparison with sympatric and allopatric raptors, Gluckman and Mundy (2013) also showed that the appearance of the underpart barring of cuckoos is more similar to that of sympatric raptors than allopatric raptors, suggesting that selection for hawk mimicry may act locally similar to that for egg mimicry.
Although both egg mimicry and hawk mimicry are likely to be based on reciprocal interactions between brood parasites and their hosts, our understanding of hawk mimicry is much less than that of egg mimicry that has placed avian brood parasitism as a representative model system of coevolutionary study (Davies, 2000; Payne and Sorensen, 2005; Trnka et al., 2012). This is partly due to the technical difficulty in collecting sufficient quantitative information on the hawk-like features of wild cuckoos that are extremely shy and secluded. As a result, contrary to the advancement of measurement in egg coloration (Brooke and Davies, 1988; Davies and Brooke, 1989; Moksnes and ØSkaft, 1995; Davies, 2000; Stokke et al., 2002; Payne and Sorensen, 2005; Stoddard and Stevens, 2010, 2011), most descriptions of hawk-like features that have appeared in recent studies or books tend to be qualitative or derived from a small number of museum specimens that were collected haphazardly in space and time, and some traits, including iris color, cannot be measured from these specimens (Payne and Sorensen, 2005; Erritzøe et al., 2012; Lehikoinen and Väisänen, 2020). Furthermore, paralleling Gluckman and Mundy's (2013) study, our knowledge of variations in hawk-like features in sympatric cuckoos of different species needs to be expanded. Such accumulation of quantitative data on hawk-like features across species and how much they vary within and among sympatric cuckoo species, would be a fundamental step to understand and generalize the hawk mimicry of cuckoos from a coevolutionary perspective.

In this study, we quantitatively compared the hawklike features within and among the four sympatric Cuculus cuckoos (the lesser cuckoo C. poliocephalus, the Indian cuckoo C. micropterus, the oriental cuckoo C. optatus, and the common cuckoo C. canorus) wild-captured in South Korea (Figure 1). Specifically, we first measured a well-recognized hawk-like feature such as eye color (i.e., colors of the eye ring and iris) and the thickness of the underpart barring across species. We also compared other potential hawk-like features that have been rarely quantified in previous studies, such as inner wing spots and underpart background colors, as well as traditional morphometric traits, including body mass and wing length. We then discuss potential reasons that generate variations in hawk-like features within and among species, and suggest future directions to increase our understanding of visual signals in avian brood parasitism.

\section{MATERIALS AND METHODS}

\section{Fieldwork}

Fieldwork to collect morphological data of wild cuckoos was conducted across the South Korea during the breeding season (May-July) between 2015 and 2020. We captured the four species of cuckoos belonging to the genus Cuculus using mist nets with song playback and dummy cuckoos mimicking the respective species (Figure 1). We captured 28 oriental cuckoos (21 male and seven female), 68 common cuckoos (56 male and 12 female), and three Indian cuckoos (two male and one female) in and around Yangpyeong-gun $\left(37^{\circ} 29 \mathrm{~N}, 127^{\circ} 29 \mathrm{E}\right)$ between 2018 and 2020. Of the lesser cuckoo, which mostly occurs in the southern part of 

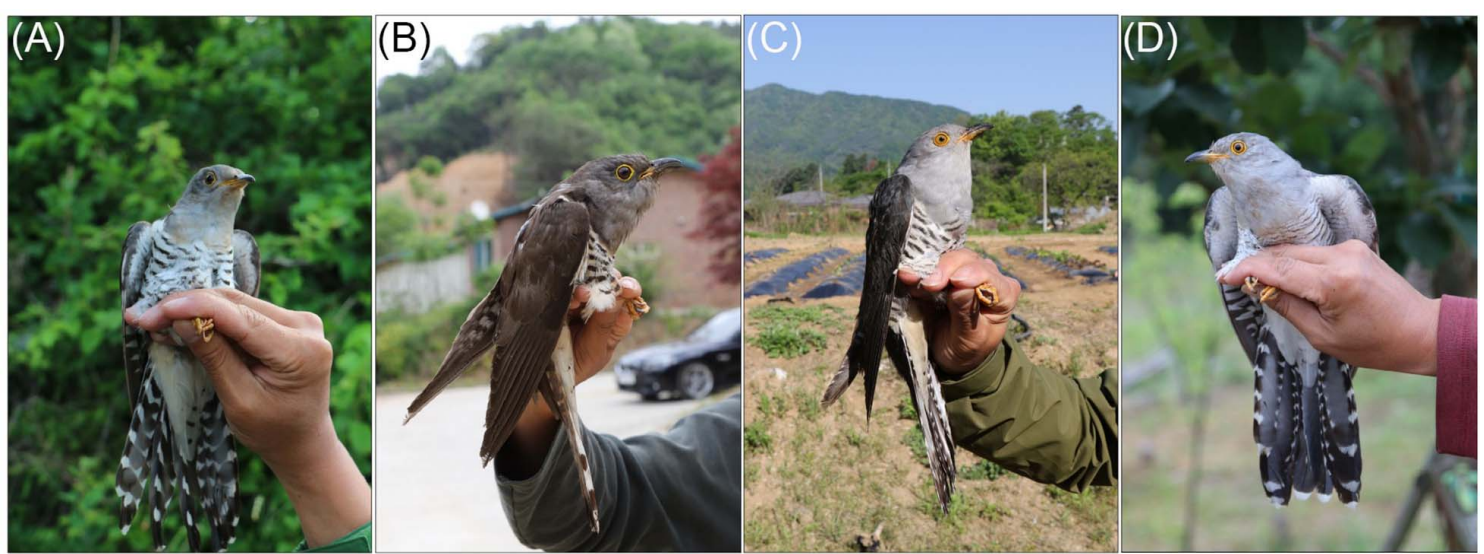

FIGURE 1 | Photographs of the four Cuculus cuckoos: (A) male lesser cuckoo, C. poliocephalus, (B) male Indian cuckoo, C. micropterus, (C) male oriental cuckoo, C. optatus, (D) male common cuckoo, C. canorus. @All photos, J-W Lee.

TABLE 1 | Mean ( \pm s.d.) body size, number of inner wing spots, and thickness of underpart barring of the four Cuculus male cuckoos.

\begin{tabular}{|c|c|c|c|c|c|c|c|}
\hline & Lesser cuckoo & Indian cuckoo & Oriental cuckoo & Common cuckoo & $\boldsymbol{F}$ & $d f$ & $p$ \\
\hline Wing & $160.2 \pm 4.10(39)^{a}$ & $215.3 \pm 7.42(2)$ & $201.7 \pm 4.49(21)^{b}$ & $214.8 \pm 5.24(56)^{c}$ & 1554.0 & 2,113 & $<0.05$ \\
\hline Tarsus & $19.2 \pm 0.46(37)^{a}$ & $23.0 \pm 0.78(2)$ & $21.7 \pm 0.66(21)^{b}$ & $22.5 \pm 0.71(56)^{c}$ & 312.9 & 2,111 & $<0.05$ \\
\hline Head-bill & $45.7 \pm 0.85(39)^{a}$ & $58.3 \pm 2.90(2)$ & $53.7 \pm 1.27(21)^{b}$ & $53.2 \pm 1.40(56)^{b}$ & 511.0 & 2,113 & $<0.05$ \\
\hline Bill length & $16.8 \pm 0.47(37)^{a}$ & $23.3 \pm 0.85(2)$ & $20.1 \pm 0.57(21)^{b}$ & $20.0 \pm 0.70(56)^{b}$ & 338.3 & 2,111 & $<0.05$ \\
\hline Bill Depth & $7.0 \pm 0.24(39)^{a}$ & $10.8 \pm 0.92(2)$ & $9.0 \pm 0.32(21)^{b}$ & $8.6 \pm 0.45(56)^{c}$ & 285.6 & 2,113 & $<0.05$ \\
\hline Wing spots & $19.7 \pm 1.33(28)^{a}$ & $18.0 \pm 1.41(2)$ & $18.9 \pm 1.45(16)^{a}$ & $24.0 \pm 1.74(49)^{b}$ & 102.2 & 2, 91 & $<0.05$ \\
\hline Barring & $2.1 \pm 0.21(19)^{a}$ & $4.1 \pm 0.15(2)$ & $2.4 \pm 0.26(18)^{b}$ & $1.5 \pm 0.24(48)^{c}$ & 122.2 & 2,84 & $<0.05$ \\
\hline
\end{tabular}

Length and thickness are measured at millimeter level $(\mathrm{mm})$ and mass at gram $(\mathrm{g})$. Numbers in the parentheses represent sample size (i.e., the number of individual measured). The results of the one-way ANOVA test from which the Indian cuckoo is excluded are provided. Different superscript letters on the values indicate statistically significant differences from post hoc tests.

TABLE 2 | Mean ( \pm s.d.) body size, number of inner wing spots, and thickness of underpart barring of the four Cuculus female cuckoos.

\begin{tabular}{|c|c|c|c|c|c|c|c|}
\hline & Lesser cuckoo & Indian cuckoo & Oriental cuckoo & Common cuckoo & $\boldsymbol{F}$ & $d f$ & $p$ \\
\hline Mass & $59.2 \pm 3.02(17)^{a}$ & $85.5(1)$ & $87.2 \pm 2.70(7)^{b}$ & $94.5 \pm 4.96(12)^{C}$ & 350.1 & 2,33 & $<0.05$ \\
\hline Wing & $156.8 \pm 3.09(18)^{a}$ & $211.5(1)$ & $186.4 \pm 5.47(7)^{b}$ & $205.6 \pm 6.94(12)^{c}$ & 345.0 & 2,34 & $<0.05$ \\
\hline Tail & $130.8 \pm 4.87(18)^{a}$ & $169(1)$ & $151.4 \pm 4.61(7)^{b}$ & $158.3 \pm 2.71(10)^{c}$ & 148.1 & 2,32 & $<0.05$ \\
\hline Tarsus & $19.6 \pm 0.42(18)^{a}$ & $22.8(1)$ & $20.6 \pm 0.44(7)^{b}$ & $22.0 \pm 0.71(12)^{c}$ & 75.4 & 2,34 & $<0.05$ \\
\hline Head-bill & $45.6 \pm 0.87(18)^{a}$ & $59(1)$ & $51.3 \pm 1.24(7)^{b}$ & $51.3 \pm 1.49(12)^{b}$ & 110.8 & 2,34 & $<0.05$ \\
\hline Bill length & $17.0 \pm 0.59(18)^{a}$ & $23.4(1)$ & $19.0 \pm 0.75(7)^{b}$ & $19.1 \pm 0.58(12)^{b}$ & 55.9 & 2,34 & $<0.05$ \\
\hline Bill Width & $8.0 \pm 0.26(18)^{a}$ & $10.6(1)$ & $9.0 \pm 0.20(7)^{b}$ & $9.2 \pm 0.43(12)^{b}$ & 56.3 & 2,34 & $<0.05$ \\
\hline Bill Depth & $6.9 \pm 0.20(18)^{a}$ & $10(1)$ & $8.6 \pm 0.29(7)^{b}$ & $8.4 \pm 0.41(12)^{b}$ & 120.4 & 2,34 & $<0.05$ \\
\hline Wing spots & $20.9 \pm 1.34(16)^{a}$ & $19(1)$ & $20.1 \pm 2.97(7)^{a}$ & $25.4 \pm 1.83(12)^{b}$ & 24.5 & 2,32 & $<0.05$ \\
\hline Barring & $2.3 \pm 0.16(16)^{a}$ & $3.6(1)$ & $2.7 \pm 0.30(7)^{b}$ & $1.3 \pm 0.24(12)^{c}$ & 103.8 & 2,32 & $<0.05$ \\
\hline
\end{tabular}

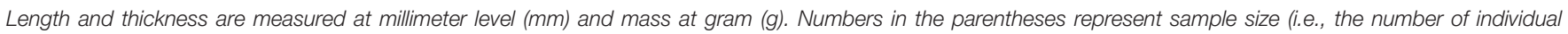

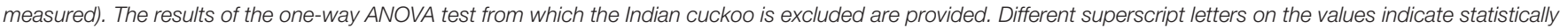
significant differences from post hoc tests.

Korea, we caught a total of 57 individuals ( 39 male and 18 female) in Jeju-do $\left(33^{\circ} 29 \mathrm{~N}, 126^{\circ} 29 \mathrm{E}\right)$ between 2015 and 2016 . The sex of the individual was determined using vocal cues confirmed in the field. After capturing, we metal-ringed each individual and measured the morphological traits related to body size, including body mass, wing length (primary feathers), tail length, tarsus length, head-bill length, and bill size (length, width, and depth from/on nostril) using standard methods (Eck et al., 2011). 


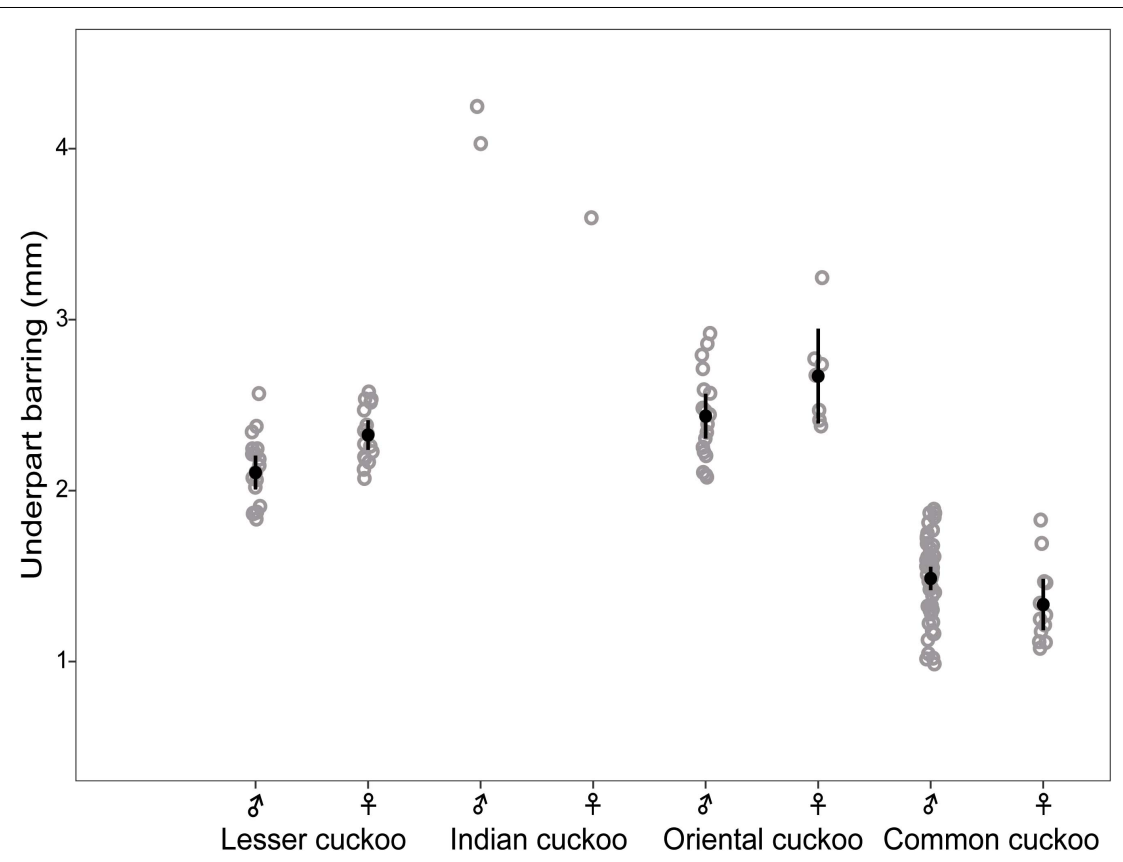

FIGURE 2 | The thickness of underpart barring of males and females of the four Cuculus cuckoos. Each gray open dot represents the thickness of different individuals, for which the thickness of three randomly selected barring were averaged. Black dots with vertical bars indicate mean values with its $95 \%$ confidence intervals.

TABLE 3 | Eye colors (iris and eye-ring) according to right/left eye, sex and species among the four Cuculus cuckoos.

\begin{tabular}{|c|c|c|c|c|c|c|c|c|c|}
\hline \multirow[t]{2}{*}{ Parts } & \multirow[t]{2}{*}{ Codes } & \multicolumn{2}{|l|}{ Lesser cuckoo } & \multicolumn{2}{|l|}{ Indian cuckoo } & \multicolumn{2}{|c|}{ Oriental cuckoo } & \multicolumn{2}{|c|}{ Common cuckoo } \\
\hline & & Male (16) & Female (15) & Male (2) & Female (1) & Male (15) & Female (6) & Male (33) & Female (9) \\
\hline \multicolumn{10}{|l|}{ Right eye } \\
\hline \multirow[t]{2}{*}{ Iris } & RGB code & $(85,67,47)$ & $(91,72,46)$ & $(46,37,32)$ & $(41,31,24)$ & $(132,90,26)$ & $(143,119,76)$ & $(184,134,22)$ & $(185,148,64)$ \\
\hline & Hex code & \#55432F & \#5B482E & \#2E2520 & \#291F18 & \#845A1A & \#8F774C & \#B88616 & \#B99440 \\
\hline \multirow[t]{2}{*}{ Eye-ring } & RGB code & $(236,199,7)$ & $(235,203,19)$ & $(230,197,10)$ & $(234,206,4)$ & $(229,180,6)$ & $(216,189,26)$ & $(236,194,3)$ & $(234,209,8)$ \\
\hline & Hex code & \#ECC707 & \#EBCB13 & \#E6C50A & \#EACE04 & \#E5B406 & \#D8BD1A & \#ECC203 & \#F3D108 \\
\hline \multicolumn{10}{|l|}{ Left eye } \\
\hline \multirow[t]{2}{*}{ Iris } & RGB code & $(88,72,50)$ & $(89,70,46)$ & $(50,36,31)$ & $(41,32,24)$ & $(131,91,26)$ & $(132,112,67)$ & $(180,129,25)$ & $(181,144,58)$ \\
\hline & Hex code & \#584832 & \#59462E & \#32241F & \#292018 & \#835B1A & \#847043 & \#B48119 & \#B5903A \\
\hline \multirow[t]{2}{*}{ Eye-ring } & RGB code & $(241,205,15)$ & $(242,208,28)$ & $(254,218,18)$ & $(247,213,0)$ & $(228,180,11)$ & $(207,180,30)$ & $(240,197,8)$ & $(231,203,6)$ \\
\hline & Hex code & \#F1CD0F & \#F2D01C & \#FEDA12 & \#F7D500 & \#E4B40B & \#CFB41E & \#F0C508 & \#Е7CB06 \\
\hline
\end{tabular}

Representative colors are presented by RGB and Hex color code, for which the RGB values of individuals in the parentheses were averaged.

We also took images of each cuckoo using a digital camera (Canon EOS 70D) with a standard color chart (X-rite ColorChecker Passport) placed beside each individual for color or size calibration, from which we obtained data on eye color, underpart barring thickness, the number of inner wing spots, and underpart color. Using the Capture One 20 software from Phase One (Erni, 2017), we quantified eye colors from photos, for which we extracted the RGB color values from five randomly selected points in the area of the iris (excluding the iris flecking area, see Yoo et al., 2017) and eye ring, respectively, after which an average of the values was taken. We also provided hex color code (e.g., \#2E231D) for the corresponding RGB values to facilitate color checking, where the first two digits after \# represent the $R$ value, the next two and the last two digits represent the $G$ and $B$ value, respectively. Although ultraviolet (UV) vision in birds are well recognized and thus measuring colors including UV light as well as visible lights is common place (Finger and Burkhardt, 1994; Hausmann et al., 2003; Stevens and Cuthill, 2007; Stoddard, 2012; Tedore and Nilsson, 2019), the application of these technologies was limited in this study due to logistical constraints. We also measured the thickness of the underpart barring using ImageJ ver. 1.52a (Schneider et al., 2012), for which we randomly selected three barrings located on the upper belly. The number of inner wing spots was determined by counting all spots observed on the three out primaries $(\mathrm{p} 9=$ second-out primaries, $\mathrm{p} 8=$ thirdout primaries, $\mathrm{p} 7$ = fourth-out primaries) of the right wing. The 

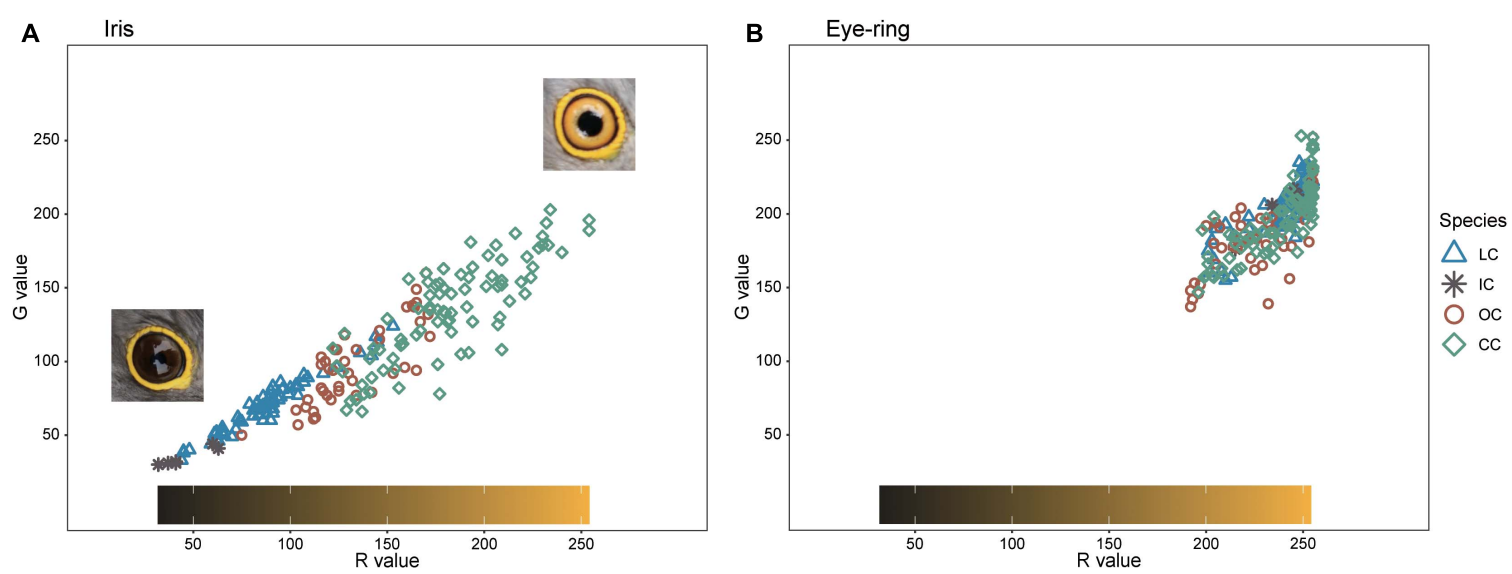

FIGURE 3 | The $R$ and $G$ values of iris (A) and eye-ring color (B) in the four Cuculus cuckoos. Among the RGB color code, for visual understanding, only $R$ and $G$ values are represented. The color bar in the box represents the approximate color of iris and eye-ring of the observed combination of $R$ and $G$ values. The shapes and colors of dots are different according to species. LC, lesser cuckoos; IC, Indian cuckoos; OC, oriental cuckoos; CC, common cuckoos.
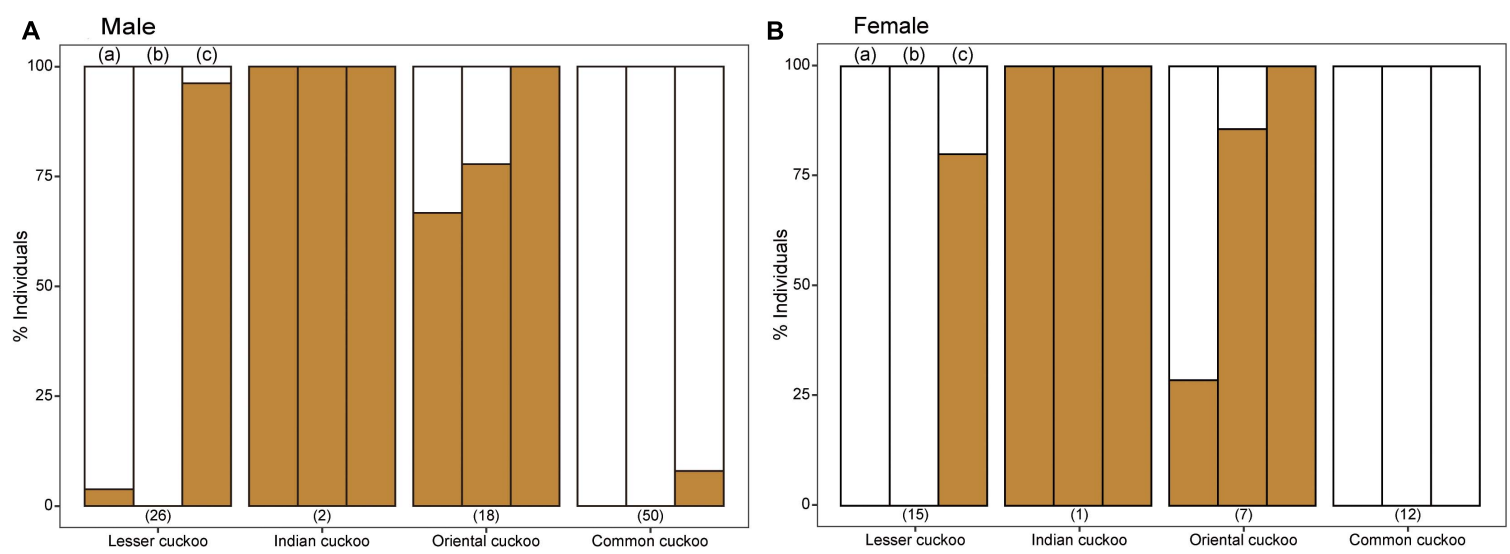

FIGURE 4 | The relative proportion of the four Cuculus cuckoos according to their underpart background colors: either pure white or yellowish-brown tinge. The colors of different areas of underpart [i.e., (a) belly, (b) underwing covert, and (c) undertail covert] were accessed separately. The numbers in the parentheses represent the sample size. (A) Male Cuculus cuckoos, (B) Female Cuculus cuckoos.

background colors of the underpart (i.e., belly, underwing covert, and undertail covert) were scored binomially either 0 (white) or 1 (yellowish brown) from the photo.

\section{Statistical Analyses}

All data except those of the Indian cuckoo met the condition of normality. We first conducted classical tests (e.g., the Student's $t$-test for sexual comparison, ANOVA for species difference) to provide information on the quantitative comparison of morphology data in the four Cuculus cuckoos. Due to the small sample size, however, the Indian cuckoo was excluded from those analyses. Tukey HSD test was applied as a post hoc test for significant differences from the ANOVA. Secondly, to test morphological differences more statistically, we carried out a principal component analysis (PCA) where body mass, wing length, tail length, bill length and head-bill length, the thickness of the underpart barring, the $R, G, B$ value of the iris, and number of inner wing spots of the four species of cuckoos were included. We then adopted the first two principal components (PCs) with eigenvalues $>1$, with which we constructed a linear model (i.e., ANOVA). PCs were used as response variables and sex and species were included as explanatory variables in the model. We achieved a minimal adequate model by removing non-significant terms until all terms in the model were significant (Crawley, 2013). All statistical tests were conducted using the $\mathrm{R}$ version 4.0.2 (R Core Team, 2020).

\section{RESULTS}

Among the four Cuculus cuckoos that regularly breed in South Korea, the Indian cuckoo was the largest in general and the lesser cuckoo was the smallest. However, the order could be changed depending on the traits measured and sex (Tables 1, 2). For example, although male common cuckoos were larger than male oriental cuckoos and similar to Indian cuckoos when it came 


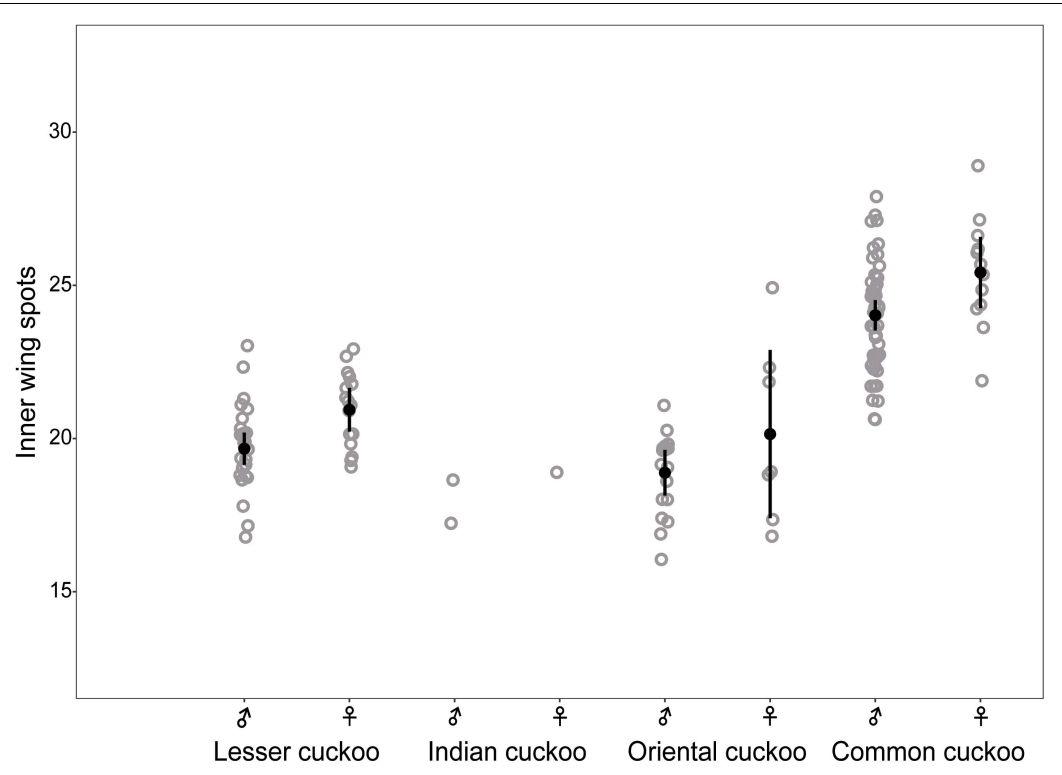

FIGURE 5 | Comparison of the number of inner wing spots according to sex and species among the four Cuculus cuckoos. Each dot indicates the total number of white spots located on the three primaries $(p 9,8,7)$ of the right wing. Black dots with vertical bars indicate mean values with its $95 \%$ confidence intervals.

to wing length, these two latter cuckoos were much heavier than male common cuckoos (Table 1). In females, however, common cuckoos were heavier and at the same time had longer primaries than oriental cuckoos (Table 2). In fact, female common cuckoos were heaviest among female Cuculus cuckoos, resulting in smaller sexual dimorphism with respect to body mass, than those in the Indian cuckoo and oriental cuckoo (Tables 1, 2).

Barred underparts are one of the key hawk-like features, and we found that the thickness of the underpart barring varied among species (Tables 1, 2 and Figure 2). In males, for example, the Indian cuckoo had the thickest barring (on average $c a .4 .1 \mathrm{~mm})$, followed by the oriental cuckoo $(2.4 \mathrm{~mm})$ and the lesser cuckoo $(2.1 \mathrm{~mm})$, and the common cuckoo

TABLE 4 | Results of the principal component analysis for the 11 morphological traits of the four Cuculus cuckoos.

\begin{tabular}{lll}
\hline Variables & PC1 (72.5\%) & PC2 (19.3\%) \\
\hline Mass & 0.20 & $-\mathbf{0 . 4 2}$ \\
Wing & 0.27 & $-\mathbf{0 . 4 2}$ \\
Tail & 0.19 & $-\mathbf{0 . 3 1}$ \\
Tarsus & 0.02 & -0.02 \\
Wing spots & 0.02 & 0.00 \\
Bill & 0.01 & -0.03 \\
Head to bill & 0.03 & -0.07 \\
Barring & -0.01 & -0.00 \\
$R$ value of iris & $\mathbf{0 . 7 3}$ & 0.08 \\
G value of iris & $\mathbf{0 . 5 6}$ & 0.37 \\
$B$ value of iris & -0.01 & $\mathbf{0 . 6 3}$ \\
\hline
\end{tabular}

The percentages in parentheses indicate the amount of variation explained by each $P C$, and the components that were loaded most highly for each parameter are in bold. Total explanation power $91.8 \%$. had the thinnest barring $(1.5 \mathrm{~mm})$. In lesser cuckoos, females had thicker barring than males, but such significant sexual differences were not observed in other species. The iris color of the four Cuculus cuckoos also varied significantly among species, ranging from nearly black (average hex color code: \#2E231D) in Indian cuckoos, dark (\#58462F), and light (\#856227) brown in lesser cuckoos and oriental cuckoos, respectively, to yellow (\#B68720) in common cuckoos (Table 3). However, there were also substantial variations in the iris color among individuals of the same species, irrespective of sex, making it difficult to define a single representative color for the iris (Figure 3). For example, some common cuckoos had bright yellow irises, while others were nearly dark brown. Likewise, some oriental cuckoos had deep dark brown iris, in contrast, others were deep yellow. As a result, these individual variations caused large overlaps in iris color between species, as shown between oriental cuckoos and common cuckoos (Figure 3). In contrast, the eye-ring color of the four Cuculus cuckoos generally represented a yellow color with a relatively small variation between species as well as between individuals of the same species (Figure 3). The brightest yellow (\#FFFC23) was observed in the common cuckoo, and the darkest (\#C08901) was observed in the oriental cuckoo (Figure 3).

The four Cuculus cuckoos also varied in the background color of their underparts, including belly, underwing, and undertail coverts (Figure 4). Both male and female common cuckoos represented a pure white color across their underparts, whereas most oriental cuckoos and Indian cuckoos had a brownish tinge as a background color. The lesser cuckoos also represented a pale yellowish-brown tinge in their undertail coverts. The total number of inner wing spots presented in the second, third, and fourth out primaries were approximately 20 across four Cuculus cuckoos (Figure 5). However, the number of spots was significantly larger in the common cuckoo than in the other three 


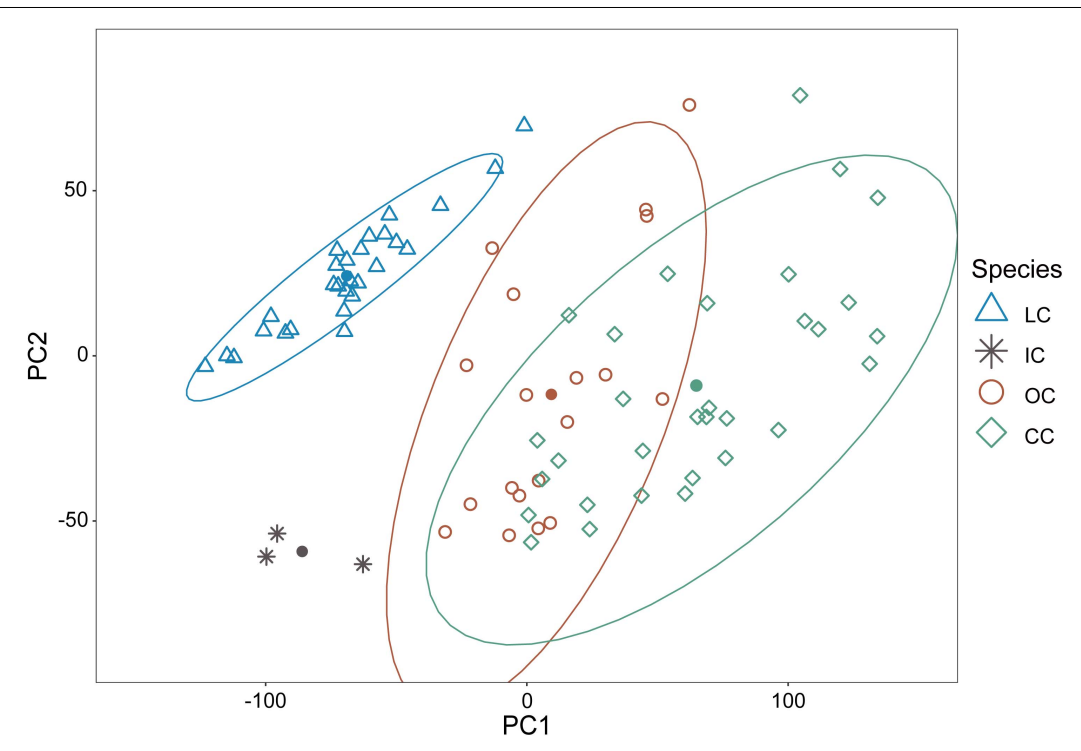

FIGURE 6 | The score plot of PC1 versus PC2 of the principal component analysis for the 11 morphology features of the four Cuculus cuckoos. PC1 explains $72.5 \%$ of the total variations while PC2 explains $19.3 \%$ of the total variations in the data. The shapes and colors of the dots are different according to species. LC, lesser cuckoos; IC, Indian cuckoos; OC, oriental cuckoos; CC, common cuckoos. Close circles are centroid vale (PC1 and PC2) of each species.

TABLE 5 | Results of the linear models for PC1 and PC2 extracted from 11 morphological traits of the four Cuculus cuckoos.

\begin{tabular}{lllll}
\hline PCs & Variables & Estimates & Std. error & $p$ \\
\hline PC1 (72.5\%) & Intercept (Lesser cuckoo) & -68.959 & 6.444 & $<0.0001$ \\
& Indian cuckoo & -17.051 & 20.714 & 0.413 \\
& Oriental cuckoo & 78.319 & 10.135 & $<0.0001$ \\
& Common cuckoo & 133.830 & 8.890 & $<0.0001$ \\
& Sex & 9.068 & 8.221 & 0.273 \\
PC2 (19.3\%) & Intercept (Lesser cuckoo) & 22.614 & 5.303 & $<0.0001$ \\
& Indian cuckoo & -84.539 & 15.462 & $<0.0001$ \\
& Oriental cuckoo & -55.902 & 7.783 & $<0.0001$ \\
& Common cuckoo & -44.718 & 6.760 & $<0.0001$ \\
& Sex & 3.460 & 7.783 & 0.658 \\
& Indian cuckoo: sex & 4.665 & 26.332 & 0.860 \\
& Oriental cuckoo: sex & 64.997 & 12.781 & $<0.0001$ \\
& Common cuckoo: sex & 54.599 & 11.765 & $<0.0001$
\end{tabular}

Response variables are species, sex, and their interactions. The results of interaction terms for PC1 which are all non-significant are not presented for simplicity. Sex refers to the difference from male.

species, and females had more spots than males across species with variable statistical significance (Figure 5 and Tables 2, 3).

Collectively, the results of PCA showed that the morphological variations of cuckoos were explained by the first two PCs, which explained $91.8 \%$ of the total variance in the data (Table 4). PC1 best explained the variation of a hawk-like feature, the iris color ( $R$ and $G$ value), which explained $72.5 \%$ of the total variation in the data. PC2 was associated with body size, including mass, wing length, tail length, and iris color ( $B$ value), which accounted for $19.3 \%$ of the total variation. Increasing PC1 and PC2 values represented more yellow eyes and smaller body sizes. Meanwhile, the explanatory powers of other features, such as tarsus, wing spots, bill, head to bill, and barring thickness were low in this dataset (Table 4). The score plot for the first two PCs showed that PC1 was spread widely along the axis according to species (Figure 6) and the linear model showed that those of species differences were statistically significant (Table 5 and Figure 7). In PC2 the values showed small but distinct variations among species as well as sex in the Oriental and common cuckoos (Table 5 and Figures 6, 7). Overall, the plot showed that the lesser cuckoo and Indian cuckoo were clearly distinct from each other and from the common cuckoo and oriental cuckoo. The oriental cuckoo and common cuckoo almost overlapped in PC2 and some overlapped in PC1, making them most similar in appearance among the four species (Figures 6, 7).

\section{DISCUSSION}

Our study shows that the four sympatric Cuculus cuckoos share an overall hawk-like appearance, such as yellow eyes and feet, gray upperparts, and pale barred underparts, which often make it difficult to distinguish them in the field (Lehikoinen and Väisänen, 2020). However, the detailed attributes of these features varied considerably between species. First, previous experimental studies proposed the importance of underpart barring as a hawklike feature in the common cuckoo (Davies and Welbergen, 2008; Welbergen and Davies, 2011). However, this species had the thinnest barring among the four Cuculus cuckoos considered in this study. Second, the yellow eyes (yellow iris and eyering) of the common cuckoo have been considered as hawklike features (Thorogood and Davies, 2013), as well as cues for species recognition in some host species (Trnka et al., 2012). However, such bright yellow eyes were seldom observed in the other three species. These species had nearly black or brown 

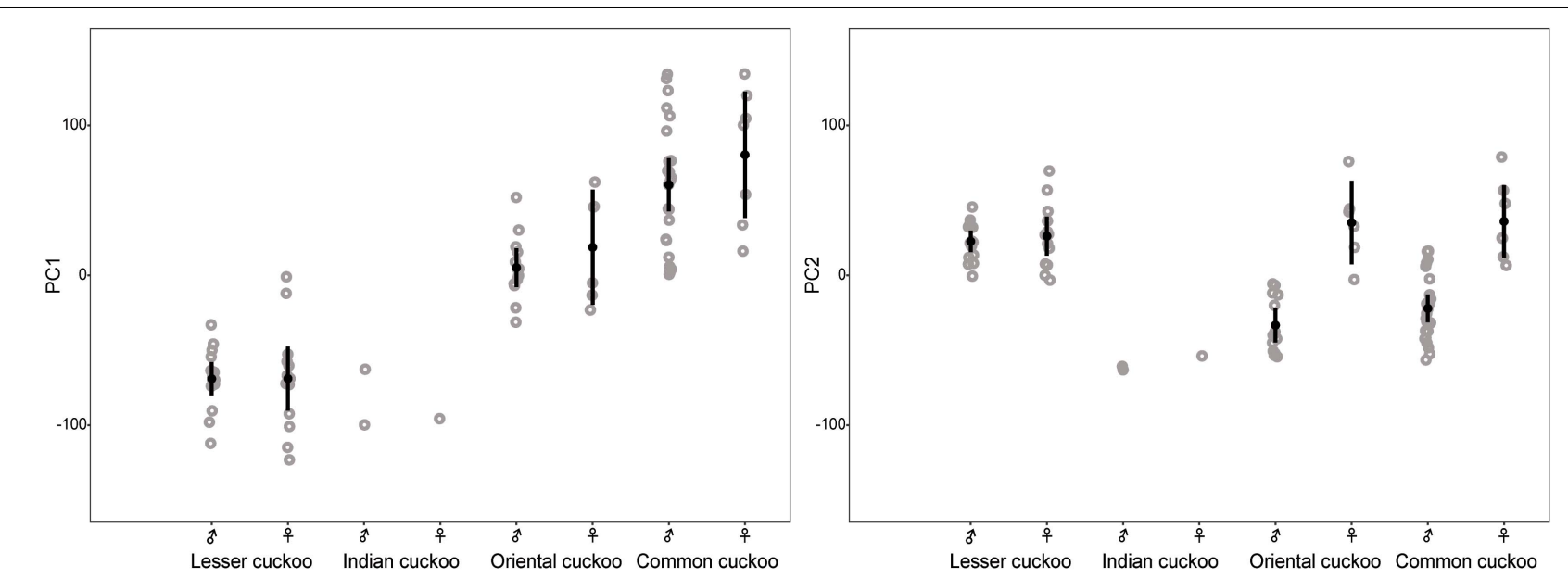

FIGURE 7 | Comparison of the PC1 and PC2 among the four Cuculus cuckoos. Each dot indicates the value of PC1 and PC2. Black dots with vertical bars indicate mean values with its $95 \%$ confidence intervals.

iris, although the color of the eye ring was yellow with small variations, indicating that the color of the iris and eye-ring may be determined by different biochemical and genetic pathways. For example, in some species, the eye-ring color is known to be carotenoid-based, so their color is largely affected by the food they consume (Bortolotti et al., 2003; Pérez-Rodríguez and Viñuela, 2008) and similarly the eye-ring color of cuckoos reared in captivity become paler, probably due to change in food (Meshcheryagina and Opaev, 2021). In contrast, the iris color may be determined by multiple agents, including various types of pigments, such as melanin and purines (Oliphant, 1987; Waldvogel, 1990; Sweijd and Craig, 1991; Gill and Prum, 2007), patterns including flecking, superficial blood vessels, and eye structures, irrespective of pigmentation, and also vary according to age, sex, and social status (Newton and Marquiss, 1982; Sweijd and Craig, 1991; Scholten, 1999; Bortolotti et al., 2003; Volpato et al., 2003; Guzzetti et al., 2008). In this study, however, we did not measure UV light which birds including host species are able to see. Future studies that measure eye color under UV vision are necessary to fully generalize the species difference in eye color in cuckoos. Third, although the common cuckoo has an elongated body with a long tail and wings and a lighter body mass, generating a hawk-like flight shape, such flight features seem to be less clear, at least in the Indian cuckoo, which has a shorter tail but heavier body mass, leading to fast but waddling wing flapping compared to the common cuckoo. Overall, these results show that although their respective host species meet the same dangerous raptor species in the area, the hawk-like features of sympatric cuckoos of different species could vary in their attributes. Whether these interspecific variations in hawklike features are derived as a result of different host responses remains to be tested in future studies.

Although the four cuckoo species could occur in the same area, specific habitat preferences may differ among species (Lee et al., 2014). For example, both the oriental cuckoo and Indian cuckoo appear to prefer mountainous areas, whereas the common cuckoo and the lesser cuckoo have broader habitat preferences, including open areas such as grasslands, reedbeds, and mountains (del Hoyo et al., 1997; Payne and Sorensen, 2005; Allen et al., 2012; Erritzøe et al., 2012; Lee et al., 2014; Yun et al., 2020). Therefore, these differences in habitat preferences may alternatively generate variations in their morphology (Linsdale, 1938; Hamilton, 1961; Norberg, 1979, 1990). For instance, dark iris, thick barring, and brownish underpart background color that are observed in the oriental cuckoo and Indian cuckoo may effectively increase their degree of camouflage in dark habitats such as forests, thus increasing the chance of accessing host nests, and also protecting them from predatory attacks (Lindholm and Lindén, 2003; Speed et al., 2005; Rowland, 2009; Welbergen and Davies, 2011; Barnett et al., 2017). However, clear differences in eye color and barring thickness between the common cuckoo and the lesser cuckoo that share a similar habitat preference, may indicate that habitat structures and light conditions therein may not be the only factors causing morphological variations among species. Morphological features, including body mass and the thickness of the underpart barring, also differ among subspecies of the common cuckoo (del Hoyo et al., 1997; Payne and Sorensen, 2005; Erritzøe et al., 2012; Lehikoinen and Väisänen, 2020). As shown in the score plot, among the four Cuculus species, the common cuckoo and the oriental cuckoo that are phylogenetically closest are also most similar in appearance (Payne and Sorensen, 2005; Wang et al., 2016). Therefore, regional and/or phylogenetic effects need to be considered to comprehensively understand their morphological variations (Linsdale, 1938; Hamilton, 1961; Norberg, 1990, 1995; Thorogood and Davies, 2013; Wang et al., 2016; Qiu et al., 2019).

The Accipiter hawk generally has dark spots on whitebackground inner wings, so one looks at dark inner wing linings from the bottom (Parkes, 1955; Kuroda, 1966; Newton, 2010). In contrast, the cuckoos show a reverse pattern with white spots on dark-background inner wings (i.e., white inner wing linings). Such inner wing patterns may increase the cryptic function of camouflage while approaching prey or 
hosts (Newton, 2010; Welbergen and Davies, 2011). In addition, Lehikoinen and Väisänen (2020) showed that the common cuckoo had more spots on the primary wing than the oriental cuckoos, suggesting that the number of wing spots could be used to distinguish the common cuckoo from the oriental cuckoo. Our study further shows that the common cuckoo has more spots on the primary than the lesser cuckoo and the Indian cuckoo as well as the oriental cuckoo, thereby distinguishing the common cuckoo from the other three cuckoos by the wings, albeit not among those three. However, their number also seems to vary among individuals within a species, and likely between sexes. Further studies revealing the biological meaning of these variations and their role in hawk mimicry would be worthwhile.

Thus, this study has shown the quantitative variations in morphology including hawk-like features among the four sympatric Cuculus cuckoos. Our results suggest that comparing body size in, for example, the meta-analysis should be conducted with caution because the outcome could be changed according to traits (e.g., body weight, wing length) and sex. For hawklike features, our results also indicate that the detailed attributes of hawk-like features such as eye color, underpart barring, and flight shape could be different among sympatric cuckoo species that share the same model species locally. Comparing these inter-specific variations with those from other assemblages of sympatric cuckoos that have different model species, would be necessary to elucidate the effect of model species on the evolution of hawk mimicry among sympatric cuckoos. Furthermore, experimental studies testing the adaptability of hawk mimicry across diverse cuckoo-host systems would help generalize the hypothesis that the hawk-like features in brood parasitic cuckoos evolve as a result of reciprocal interactions between the cuckoos and their hosts to maximize their respective fitness.

\section{DATA AVAILABILITY STATEMENT}

The original contributions presented in the study are included in the article/Supplementary Material, further inquiries can be directed to the corresponding author.

\section{REFERENCES}

Allen, W. L., Baddeley, R., Cuthill, I. C., and Scott-Samuel, N. E. (2012). A quantitative test of the predicted relationship between countershading and lighting environment. Am. Nat. 180, 762-776. doi: 10.1086/6 68011

Barnett, J. B., Redfern, A. S., Bhattacharyya-Dickson, R., Clifton, O., Courty, T., Ho, T., et al. (2017). Stripes for warning and stripes for hiding: spatial frequency and detection distance. Behav. Ecol. 28, 373-381. doi: 10.1093/beheco/arw168

Bortolotti, G. R., Smits, J. E., and Bird, D. M. (2003). Iris colour of American kestrels varies with age, sex, and exposure to PCBs. Physiol. Biochem. Zool. 76, 99-104. doi: $10.1086 / 345485$

Brooke, M. D. L., and Davies, N. B. (1988). Egg mimicry by cuckoos Cuculus canorus in relation to discrimination by hosts. Nature 335, 630-632. doi: $10.1038 / 335630 \mathrm{a} 0$

Crawley, M. J. (2013). The R Book, 2nd Edn. Chichester: John Wiley \& Sons.

\section{ETHICS STATEMENT}

The capture of the four Cuculus cuckoos was authorized by the local governments and the cultural heritage administration of Korea. The fieldwork procedures were compiled with the national law of Korea, and the animal study was reviewed and approved by the Kyung Hee University Animal Ethics Committee.

\section{AUTHOR CONTRIBUTIONS}

J-WL conceived and designed the study. J-SG and J-WL analyzed the data. J-SG wrote the manuscript with assistance from J-WL. All authors performed the fieldwork, and reviewed and approved the final manuscript.

\section{FUNDING}

This work was financially supported by the National Research Foundation of Korea (to J-WL, grant numbers: NRF- 2017R1D1A1B03030329, 2019K2A9A2A06022677, and 2020R111A2063567).

\section{ACKNOWLEDGMENTS}

We are most grateful to many students and field assistants who joined the long-term fieldwork to collect morphological data from wild cuckoos: Geun-Won Bae, Myeong-Chan Cha, Sei-Woong Choi, Sue-Jeong Jin, Won-Ju Jung, Hae-Ni Kim, Hee-Young Kim, Kyoung-Hoe Kim, Myun-Sik Kim, Kyung-Gyu Lee, Hyeon-A Lee, Ki-Baek Nam, Hee-Jin Noh, Hong-Sik Oh, Jeong-Eun Park, Ha-Na Yoo, Sohyeon Yoo, and Seongho Yun.

\section{SUPPLEMENTARY MATERIAL}

The Supplementary Material for this article can be found online at: https://www.frontiersin.org/articles/10.3389/fevo.2021. 702263/full\#supplementary-material

Davies, N. B. (2000). Cuckoos, Cowbirds and other Cheats. London: T \& A D Poyser Ltd.

Davies, N. B., and Brooke, M. D. L. (1989). An experimental study of co-evolution between the cuckoo, Cuculus canorus, and its hosts. I. Host egg discrimination. J. Anim. Ecol. 58, 207-224. doi: 10.2307/4995

Davies, N. B., and Welbergen, J. A. (2008). Cuckoo-hawk mimicry? An experimental test. Proc. R. Soc. B. 275, 1817-1822. doi: 10.1098/rspb.2008.0331

Davies, N. B., and Welbergen, J. A. (2009). Social transmission of a host defense against cuckoo parasitism. Science 324, 1318-1320. doi: 10.1126/science. 1172227

del Hoyo, J., Elliott, A., and Sargatal, J. (1997). Handbook of the Birds of the World. Sandgrouse to Cuckoos, Vol. 4. Barcelona: Lynx Edicions.

Duckworth, J. W. (1991). Responses of breeding reed warblers Acrocephalus scirpaceus to mounts of sparrowhawk Accipiter nisus, cuckoo Cuculus canorus and jay Garrulus glandarius. Ibis 133, 68-74. doi: 10.1111/j.1474-919x.1991. tb04812.x 
Dyrcz, A., and Hałupka, L. (2006). Great reed warbler Acrocephalus arundinaceus and reed warbler Acrocephalus scirpaceus respond differently to cuckoo dummy at the nest. J. Ornithol. 147, 649-652. doi: 10.1007/s10336-006-0097-x

Eck, S., Fiebig, J., Fiedler, W., Heynen, I., Nicolai, B., Töpfer, T., et al. (2011). Measuring Birds-Vögel Vermessen. Wilhelmshaven: Deutche OrnithologenGesellschaft.

Erni, S. (2017). Capture One Pro 10: Mastering Raw Development, Image Processing, and Asset Management. San Rafael, CA: Rocky Nook, Inc.

Erritzøe, J., Mann, C. F., Brammer, F. P., and Fuller, R. A. (2012). Cuckoos of the World. London: Christopher Helm.

Finger, E., and Burkhardt, D. (1994). Biological aspects of bird colouration and avian colour vision including ultraviolet range. Vision Res. 34, 1509-1514. doi: 10.1016/0042-6989(94)90152-x

Friedmann, H. (1928). The origin of host specificity in the parasitic habit in the Cuculidae. Auk 45, 33-38. doi: 10.2307/4075354

Gill, F. B., and Prum, R. O. (2007). Ornithology, 4th Edn. New York, NY: W. H. Freeman.

Gluckman, T. L., and Mundy, N. I. (2013). Cuckoos in raptors' clothing: barred plumage illuminates a fundamental principle of Batesian mimicry. Anim. Behav. 86, 1165-1181. doi: 10.1016/j.anbehav.2013. 09.020

Guzzetti, B. M., Talbot, S. L., Tessler, D. F., Gill, V. A., and Murphy, E. C. (2008). Secrets in the eyes of Black Oystercatchers: a new sexing technique. J. Field. Ornithol. 79, 215-223. doi: 10.1111/j.1557-9263.2008.00 167.x

Hamilton, T. H. (1961). The adaptive significances of intraspecific trends of variation in wing length and body size among bird species. Evolution 15, 180-195. doi: 10.2307/2406079

Hausmann, F., Arnold, K. E., Marshall, N. J., and Owens, I. P. (2003). Ultraviolet signals in birds are special. Proc. R. Soc. B. 270, 61-67. doi: 10.1098/rspb.2002. 2200

Honza, M., Šicha, V., Procházka, P., and Ležalová, R. (2006). Host nest defense against a color-dimorphic brood parasite: great reed warblers (Acrocephalus arundinaceus) versus common cuckoos (Cuculus canorus). J. Ornithol. 147, 629-637. doi: 10.1007/s10336-006-0088-y

Krüger, O., Davies, N. B., and Sorenson, M. D. (2007). The evolution of sexual dimorphism in parasitic cuckoos: sexual selection or coevolution? Proc. R. Soc. B. $274,1553-1560$. doi: $10.1098 / \mathrm{rspb} .2007 .0281$

Kuroda, N. (1966). On the origin of raptor-pattern and hawk-mimicry of cuckoos. J. Yamashina Inst. Ornithol. 4, 384-387. doi: 10.3312/jyio195 2.4 .384

Lee, J. W., Noh, H. J., Lee, Y., Kwon, Y. S., Kim, C. H., and Yoo, J. C. (2014). Spatial patterns, ecological niches, and interspecific competition of avian brood parasites: inferring from a case study of Korea. Ecol. Evol. 4, 3689-3702. doi: 10.1002/ece3.1209

Lehikoinen, P., and Väisänen, R. J. D. B. (2020). Identification of oriental cuckoo and common cuckoo based on primary pattern. Dutch Bird. 42, 229-247.

Lindholm, A., and Lindén, A. J. A. (2003). Oriental cuckoo in Finland. Alula 8, 122-133.

Linsdale, J. M. (1938). Bird life in Nevada with reference to modifications in structure and behavior. Condor 40, 173-180. doi: 10.2307/1363426

Ma, L., Yang, C., and Liang, W. (2018). Hawk mimicry does not reduce attacks of cuckoos by highly aggressive hosts. Avian. Res. 9, 1-7.

Medina, I., and Langmore, N. E. (2016). The evolution of host specialisation in avian brood parasites. Eco. Lett. 19, 1110-1118. doi: 10.1111/ele.12649

Meshcheryagina, S. G., and Opaev, A. (2021). Previously unknown behavior in parasitic cuckoo females: male-like vocalization during migratory activity. Avian Res. 12, 1-16. doi: 10.1016/j.physbeh.2018.03.023

Moksnes, A., and ØSkaft, E. R. (1995). Egg-morphs and host preference in the common cuckoo (Cuculus canorus): an analysis of cuckoo and host eggs from European museum collections. J. Zool. 236, 625-648. doi: 10.1111/j.1469-7998. 1995.tb02736.x

Newton, I. (2010). The Sparrowhawk. London: T \& A D Poyser Ltd.

Newton, I., and Marquiss, M. (1982). Eye colour, age and breeding performance in Sparrowhawks Accipiter nisus. Bird Study 29, 195-200. doi: 10.1080/ 00063658209476757

Norberg, U. M. (1979). Morphology of the wings, legs and tail of three coniferous forest tits, the goldcrest, and the treecreeper in relation to locomotor pattern and feeding station selection. Philos. Trans. R. Soc. Lond. B. 287, 131-165. doi: 10.1098/rstb.1979.0054

Norberg, U. M. (1990). Vertebrate Flight. Berlin: Springer-Verlag.

Norberg, U. M. (1995). How a long tail and changes in mass and wing shape affect the cost for flight in animals. Funct. Ecol. 9, 48-54. doi: 10.2307/239 0089

Oliphant, L. W. (1987). Pteridines and purines as major pigments of the avian iris. Pigment. Cell. Res. 1, 129-131. doi: 10.1111/j.1600-0749.1987.tb00401.x

Parkes, K. C. (1955). Notes on the molts and plumages of the sparrow hawk. Wilson bull. 67, 194-199.

Payne, R. B. (1967). Interspecific communication signals in parasitic birds. Am. Nat. 101, 363-375. doi: 10.1086/282504

Payne, R. B. (1977). The ecology of brood parasitism in birds. Annu. Rev. Ecol. Syst. 8, 1-28. doi: 10.1007/978-3-319-73138-4_1

Payne, R. B., and Sorensen, M. D. (2005). The Cuckoos, Vol. 15. Oxford: Oxford University Press.

Pérez-Rodríguez, L., and Viñuela, J. (2008). Carotenoid-based bill and eye ring coloration as honest signals of condition: an experimental test in the redlegged partridge (Alectoris rufa). Naturwissenschaften 95, 821-830. doi: 10 . 1007/s00114-008-0389-5

Qiu, S., Liu, H., Cai, Y. S., Hou, W., Dou, L., Zhang, X. Y., et al. (2019). Complete mitochondrial genome and the phylogenetic position of the common cuckoo, Cuculus canorus bakeri (Aves: cuculiformes). Mitochondrial DNA B 4, 28022803. doi: 10.1080/23802359.2019.1660256

R Core Team. (2020). R: A Language and Environment for Statistical Computing. Version 4.0. 2. Vienna: R Foundation for Statistical Computing.

Rothstein, S. I. (1990). A model system for coevolution: avian brood parasitism. Annu. Rev. Eco. Syst. 21, 481-508. doi: 10.1146/annurev.es.21.110190.002405

Rowland, H. M. (2009). From Abbott Thayer to the present day: what have we learned about the function of countershading? Philos. Trans. R. Soc. B. Biol. Sci. 364, 519-527. doi: 10.1098/rstb.2008.0261

Schneider, C. A., Rasband, W. S., and Eliceiri, K. W. (2012). NIH Image to ImageJ: 25 years of image analysis. Nat. Methods 9, 671-675. doi: 10.1038/nmeth.2089

Scholten, C. J. (1999). Iris colour of Humboldt penguins Spheniscus humboldti. Mar. Ornithol. 27, 187-194.

Speed, M. P., Kelly, D. J., Davidson, A. M., and Ruxton, G. D. (2005). Countershading enhances crypsis with some bird species but not others. Behav. Ecol. 16, 327-334. doi: 10.1093/beheco/arh166

Stevens, M., and Cuthill, I. C. (2007). Hidden messages: are ultraviolet signals a special channel in avian communication? Bioscience 57, 501-507. doi: 10.1641/ b570607

Stoddard, M. C. (2012). Mimicry and masquerade from the avian visual perspective. Curr. Zool. 58, 630-648. doi: 10.1093/czoolo/58.4.630

Stoddard, M. C., and Stevens, M. (2010). Pattern mimicry of host eggs by the common cuckoo, as seen through a bird's eye. Proc. R. Soc. B. 277, 1387-1393. doi: 10.1098/rspb.2009.2018

Stoddard, M. C., and Stevens, M. (2011). Avian vision and the evolution of egg color mimicry in the common cuckoo. Evolution 65, 2004-2013. doi: 10.1111/ j.1558-5646.2011.01262.x

Stokke, B. G., Moksnes, A., and Røskaft, E. (2002). Obligate brood parasites as selective agents for evolution of egg appearance in passerine birds. Evolution 56, 199-205. doi: 10.1111/j.0014-3820.2002.tb00 861.x

Sweijd, N., and Craig, A. J. (1991). Histological basis of age-related changes in iris color in the African pied starling (Spreo bicolor). Auk 108, 53-59.

Tedore, C., and Nilsson, D. E. (2019). Avian UV vision enhances leaf surface contrasts in forest environments. Nat. Commun. 10, 1-12.

Thorogood, R., and Davies, N. B. (2013). Hawk mimicry and the evolution of polymorphic cuckoos. Chin. Birds 4, 39-50. doi: 10.5122/cbirds.2013. 0002

Trnka, A., and Grim, T. (2013). Color plumage polymorphism and predator mimicry in brood parasites. Front. Zool. 10:25. doi: 10.1186/1742-9994-10-25

Trnka, A., and Prokop, P. (2012). The effectiveness of hawk mimicry in protecting cuckoos from aggressive hosts. Anim. Behav. 83, 263-268. doi: 10.1016/j. anbehav.2011.10.036

Trnka, A., Prokop, P., and Grim, T. (2012). Uncovering dangerous cheats: how do avian hosts recognize adult brood parasites? PLoS One 7:e37445. doi: 10.1371/ journal.pone. 0037445 
Vepsäläinen, K. (1968). Wing length of lapwing (Vanellus vanellus) before and after skinning with remarks on measuring methods. Ornis Fenn. 45, 124-126.

Voipio, P. (1953). The hepaticus variety and the juvenile types of the cuckoo. Ornis Fenn. 30, 97-117.

Volpato, G. L., Luchiari, A. C., Duarte, C. R. A., Barreto, R. E., and Ramanzini, G. C. (2003). Eye color as an indicator of social rank in the fish Nile tilapia. Braz. J. Med. Biol. Res. 36, 1659-1663. doi: 10.1590/s0100-879x2003001200007

Waldvogel, J. A. (1990). The bird's eye view. Am. Sci. 78, 342-353.

Wallace, A. R. (1889). Darwinism: An Exponent of the Theory of Natural Selection, with Some of Its Applications (1889). Whitefish: Kessinger Publishing.

Wang, N., Liang, B., Huo, J., and Liang, W. (2016). Complete mitochondrial genome and the phylogenetic position of the Lesser Cuckoo, Cuculus poliocephalus (Aves: cuculiformes). Mitochondrial DNA A 27, 4409-4410. doi: 10.3109/19401736.2015.1089547

Welbergen, J. A., and Davies, N. B. (2009). Strategic variation in mobbing as a front line of defense against brood parasitism. Curr. Biol. 19, 235-240. doi: 10.1016/j.cub.2008.12.041

Welbergen, J. A., and Davies, N. B. (2011). A parasite in wolf's clothing: hawk mimicry reduces mobbing of cuckoos by hosts. Behav. Ecol. 22, 574-579. doi: 10.1093/beheco/arr008
Yoo, H. N., Lee, J. W., and Yoo, J. C. (2017). Asymmetry of eye color in the common cuckoo. Sci. Rep. 7:7612.

Yun, S., Lee, J. W., and Yoo, J. C. (2020). Habitat selection in the lesser cuckoo, an avian brood parasite breeding on Jeju Island, Korea. J. Ecol. Environ. 44, 1-9.

Conflict of Interest: The authors declare that the research was conducted in the absence of any commercial or financial relationships that could be construed as a potential conflict of interest.

Publisher's Note: All claims expressed in this article are solely those of the authors and do not necessarily represent those of their affiliated organizations, or those of the publisher, the editors and the reviewers. Any product that may be evaluated in this article, or claim that may be made by its manufacturer, is not guaranteed or endorsed by the publisher.

Copyright (c) 2021 Go, Lee and Yoo. This is an open-access article distributed under the terms of the Creative Commons Attribution License (CC BY). The use, distribution or reproduction in other forums is permitted, provided the original author(s) and the copyright owner(s) are credited and that the original publication in this journal is cited, in accordance with accepted academic practice. No use, distribution or reproduction is permitted which does not comply with these terms. 